\title{
Comportamiento frente a estrés hídrico de variedades nativas y especies silvestres de papa
}

\author{
M.C. Bedogni ${ }^{1}$, S. Capezio ${ }^{2}$, M. Huarte ${ }^{3}$ \\ RESUMEN
}

La sequía es una importante limitación que ejerce el medio ambiente sobre la producción de papa en las áreas tradicionales de cultivo. Ante la creciente influencia del cambio climático y debido a que la actual base genética de la papa presenta escasa variabilidad, se hace necesario identificar materiales genéticos con resistencia o tolerancia a sequía. Con el objetivo de desarrollar material genético tolerante se evaluó el comportamiento de variedades cultivadas y especies silvestres de papa. Se emplearon ocho variedades nativas cultivadas de papa y dos genotipos de Solanum tarijense (trj). Se adaptó el protocolo de ensayo de sequía propuesto por la red Latinpapa. Se realizaron tres tratamientos: Control, las plantas siguieron un esquema de riego normal; Sequía con Déficit, a partir del día 45 después de plantación (ddp) se aplicó riego con déficit, y Sequía Severa, a partir del día 45 ddp, no se aplicó riego. Hubo un efecto significativo del genotipo y del tratamiento sobre la altura de las plantas y sobre el número y el peso de tubérculos. En cuanto a número de tallos solo hubo efecto significativo sobre el genotipo. Natin Suito presentó mayor vigor en los tratamientos de sequía. Las variedades presentaron poca senescencia hasta los 87 ddp. La variedad Yema de Huevo no presentó signos evidentes de senescencia hasta los 97 ddp. Existe variabilidad entre los genotipos evaluados con respecto a su comportamiento bajo estrés hídrico. Las variedades Sipancachi, Unknown y ClS 1802 norte produjeron tubérculos bajo estrés hídrico y la variedad Unknown fue la de mayor peso de tubérculo.

Palabras Clave: estrés hídrico, tolerancia, variedades nativas de papa, Solanum tarijense

\section{Native potato varieties and wild species performance under water stress}

\begin{abstract}
Drought is an important limiting factor that the environment exerts over the potato production in traditional crop regions. In view of the influence of climatic change and the reduced genetic variability of the potato crop, it is necessary to identify genetic material with resistance or tolerance to drought. The performance of native varieties and wild species was evaluated with the objective of developing tolerant material. Eight native cultivated varieties and two trj genotipes were used. The drought trial protocol proposed in the Red LatinPapa was adapted. Three treatments were evaluated: Check, in which the plants received a normal irrigation regime, Drought with water shortage, where irrigation with water shortage was applied after the $45^{\text {th }}$ day after planting and Severe water shortage, where no irrigation was applied after 45 days. There was a significant effect of genotype and treatment on plant height, and on tuber number and weight. The number of stems was only influenced by the genotype. Natin Suito showed the
\end{abstract}

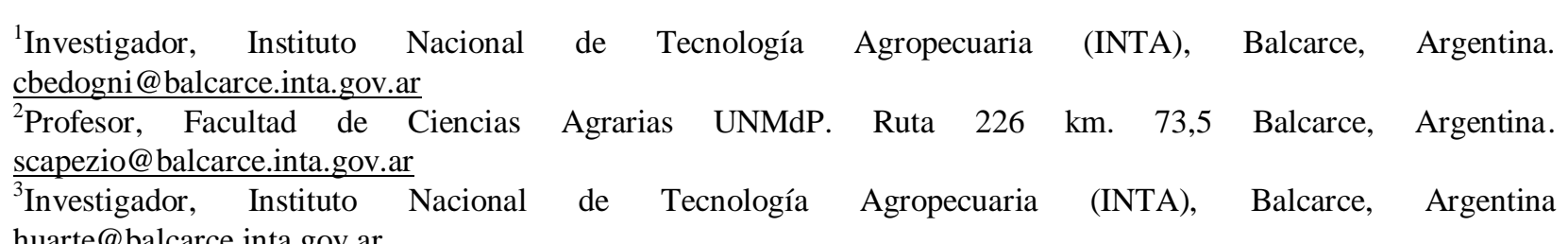


highest vigor under the drought treatments. The varieties showed little senescence up to the $87^{\text {th }}$ day after planting. The Yema de Huevo variety did not shoewed signs of senescence up to the $97^{\text {th }}$ days after planting. There is variability in performance among the varieties under water stress. Varieties Sipancachi, Unknown and CIS 1802 norte produced tubers under water stress and the variety Unknown presented the highest tuber weight.

Key words: hydric stress, tolerance, native potato, Solanum tarijense

\section{Introducción}

La sequía es una importante limitación que ejerce el medio ambiente sobre la producción de papa en las áreas tradicionales de cultivo (Ekanayake, 1993), influyendo en el desarrollo del mismo y causando pérdidas de rendimiento y calidad. Este carácter es complejo y requiere de validaciones en diversas condiciones de cultivo (Coleman, 2008).Ante la creciente influencia del cambio climático y debido a que la actual base genética de la papa presenta escasa variabilidad, se hace necesario identificar materiales genéticos con resistencia o tolerancia a sequía. De esta manera, a través de un plan de mejoramientogenético, se podría incrementar la eficiencia del uso del agua del cultivo y al mismo tiempo, permitiría aumentar el área de siembra en zonas no aptas. Es por ello que, con el objetivo de desarrollar material genético tolerante se evaluó el comportamiento de variedades cultivadas y especies silvestres de papa.

\section{Materiales y métodos}

Se emplearon ocho variedades del set común del Proyecto CYTED "Papasalud" y dos genotipos de $S$. tarijense del Banco de Germoplasma de la EEA Balcarce, Argentina (Tabla 1). Se siguió un diseño en bloques completos aleatorizados con tres repeticiones con dos plantas por repetición en invernáculo.

Tabla 1. Genotipos evaluados frente a sequía

\begin{tabular}{|c|c|}
\hline Genotipo & Especie \\
\hline Puca Quitish & Solanum tuberosum subsp. andigena (adg) \\
\hline Sipancachi & Solanum tuberosum subsp. andigena (adg) \\
\hline Puca huayro & Solanum chaucha (cha) \\
\hline Amarilla & Solanum stenotonum (sStn) \\
\hline Yema de huevo & Solanum phureja (phu) \\
\hline Poluya & Solanum stenotonum (sStn) \\
\hline Natin Suito & $\mathrm{SxG}$ \\
\hline Unknown & Solanum tuberosum subsp. andígena (adg) \\
\hline ClS 1802 norte & Solanum tarijense (trj) \\
\hline Oka $5631 x 1876 A$ & Solanum tarijense (trj) \\
\hline
\end{tabular}

Se adaptó el protocolo de ensayo de sequía propuesto por la red Latinpapa (https://research.cip.cgiar.org/confluence/display /redlatinpapa/Protocolos). Se realizaron tres tratamientos. Control (C): las plantas siguieron un esquema de riego normal, Sequía con Déficit (SD): a partir del día 45 después de plantación se aplicó riego con déficit (mitad de capacidad de campo), Sequía Severa (SS): a partir del día 45 después de plantación, no se aplicó riego). 
El vigor, la marchitez, la altura y el número de tallos de las plantas se registraron cada semana después de plantación. A la cosecha se determinaron el número, el peso, el tamaño, la uniformidad, los defectos externos e internos de los tubérculos por planta. Se realizaron análisis de varianza de las variables analizadas utilizando el programa estadístico SAS.

\section{Resultados y discusión}

Hubo un efecto significativo del genotipo y del tratamiento sobre la altura de las plantas tanto a los 45 días como a los 76 días después de plantación (ddp). Los genotipos del tratamiento control presentaron significativamente mayor altura que los tratamientos de sequía. Con respecto al número de tallos, no hubo diferencias significativas entre los tratamientos pero sí las hubo entre los genotipos. Las variedades Sipancachi, Unknown y el genotipo de trj ClS 1802 norte tuberizaron en los tres tratamientos. La variedad Amarilla no tuberizó en ningún tratamiento. Se evidenciaron diferencias significativas tanto para el número de tubérculos como para el peso de los mismos en cuanto al tratamiento aplicado como al genotipo.

Todas las plantas tuvieron relativamente alto vigor hasta el día 70 ddp. Los genotipos Unknown, Puca Huayro y Amarilla presentaron un mayor vigor en el tratamiento control y Natin Suito presentó mayor vigor en los tratamientos de sequía.

En los tratamientos control y SD las variedades presentaron poca senescencia hasta los $87 \mathrm{ddp}$.
La mayoría de los genotipos en el tratamiento de SS presentaron un gran porcentaje de mortandad luego de los 87 ddp. La variedad Yema de Huevo no presentó signos evidentes de senescencia hasta los $97 \mathrm{ddp}$.

\section{Conclusiones}

Existe variabilidad entre los genotipos evaluados con respecto a su comportamiento bajo estrés hídrico. Las variedades Sipancachi, Unknown y ClS 1802 norte produjeron tubérculos bajo estrés hídrico y la variedad Unknown fue la de mayor peso de tubérculo. Se requiere otro ciclo de ensayos en invernáculo y a campo para poder completar la caracterización frente a sequía.

\section{Literatura citada}

Centro Internacional de la Papa CIP, 2009. Experimental Protocols for Field trials assessing drought stress. En: https://research.cip.cgiar.org/confluence/display/ redlatinpapa/protocolos. (4p.). Consulta: Marzo 2010.

Coleman, W. 2008. Evaluation of wild Solanum species for drought resistance.1 Solanum gandarillasii Cardenas. Enviromental and Experimental Botany 62: 221-230.

Ekanayake, I. J. 1993. Evaluación de resistencia a la sequía en genotipos de papa y batata (camote). Guía de investigación Centro Internacional de la Papa (CIP) 19, Lima, Perú. $16 \mathrm{p}$. 